\title{
Cytomegalovirus (CMV) - a new prospect for prevention
}

\author{
Małgorzata Polz-Dacewicz', Sylwia Fołtyn' ${ }^{1}$, Paweł Macieląg ${ }^{1}$, Dorota Polz ${ }^{1}$ \\ ${ }^{1}$ Department of Virology, Medical University of Lublin \\ Polz-Dacewicz M, Fołtyn S, Macieląg P, Polz D. Cytomegalovirus (CMV) - a new prospect for prevention. J Pre-Clin Clin Res. $2013 ;$ 7(2): 118-123.
}

\begin{abstract}
Human cytomegalovirus (HCMV), a member of the Betaherpesvirinae subfamily of the Herpesviridae family, is a widespread pathogen. CMV is a virus usually completely harmless to healthy people; nevertheless, it is still a major cause of morbidity and mortality in immunocompromised individuals, such as organ transplant recipients (also haematopoietic stem cell transplantation) and AIDS patients. Furthermore, is a common cause of congenital infections. So far, it is not possible to take effective prevention activities, although there are high expectations in finding an effective vaccine. Understanding the detailed construction of the virus helped to create exactly harmless virus strains used for research. Progress in genetics and biotechnology allowed the carrying out of changes in the virus genome. Today, we know the different parts of the DNA code, are able to modify them, delete, and add inserts. All these actions bring us closer to achieving the objective of an effective and safe vaccine. The presented study shows the results of many different studies and attempts to find an effective vaccine against HCMV. The development direction of research is broad and includes various activities with the use of subunit vaccines, DNA vaccines, peptides and vaccines using the vectors. Some preparations are just at the beginning of a long journey for the registration of an acceptable vaccine, while others have entered into the last phase of clinical trials.
\end{abstract}

\section{Key words}

CMV, pathogenesis, viral tropism, therapy, vaccines

\section{INTRODUCTION}

Cytomegaloviral disease was described for the first time by Ribbert in 1881. Goodpasture and Talbert found in 1921 that this infection was caused by a viral agent. Human cytomegalovirus (HCMV) was first isolated from the cell culture by three independent researchers: Smith, Rowe (1956) and Weller (1957). The latter also gave the name of this pathogen as cytomegalovirus in 1960 [1]. HCMV is one of the more common opportunistic pathogen in immunosuppressed patients, e.g. after transplantation and $\mathrm{HIV}$ infection. Cytomegalovirus infection is especially dangerous for pregnant women because the viruses have the ability to pass through the placenta, leading to birth defects (especially in the CNS) and increase intrauterine mortality.

\section{STATE OF KNOWLEDGE}

Biology of the virus. CMV has been classified in the family Herpesviridae, subfamily Betaherpesvirinae. It is the biggest of human pathogenic viruses with a diameter of $200 \mathrm{~nm}$. It has about 200 open reading frames (ORFs) and encodes over 200 proteins. Virion has an icosahedral symmetry protein capsid consisting of 162 capsomeres, and a diameter of about $100 \mathrm{~nm}$; inside, there is a double-stranded DNA with a size of $235 \mathrm{~kb}$ [2]. The capsid is surrounded by the protein tegument and outside by the lipid coat. The viral genome consists of two covalently linked segments (L and S), each one of which contains a unique region (UL and Us) finished piece of TRL and IRL, TRs and Irs. Thus, the configuration of the genome can be represented as follows: TRL-UL-IRL-IRs-Us-TRs [2,

Address for correspondence: Małgorzata Polz-Dacewicz, Department of Virology, Medical University of Lublin

e-mail: malgorzatapolzdacewicz@umlub.pl

Received: 05 February 2014; accepted: 15 February 2014
3, 4]. The CMV genome structure is shown in Fig. 1. HCMV is routinely isolated from human fibroblast cell lines, and in studies on the biology of the virus, they are used as laboratory strains AD169, Towne, Toledo [3].

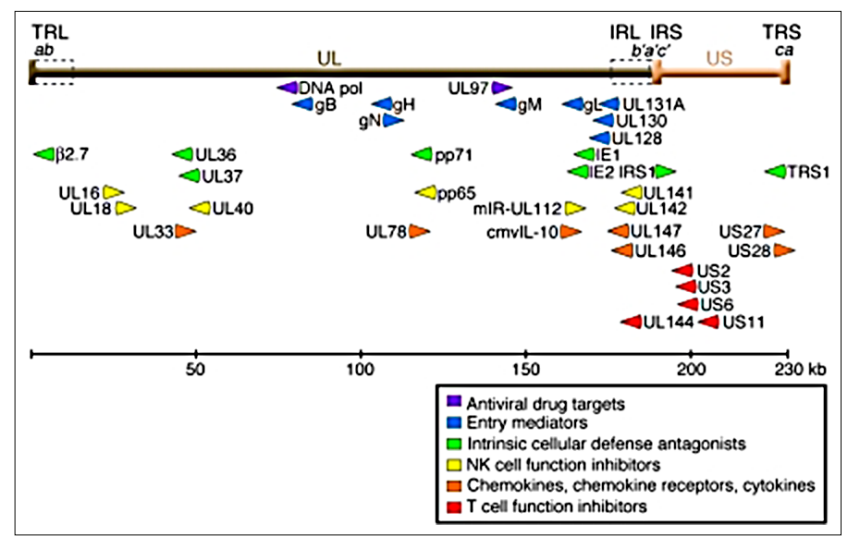

Figure 1. Structure of the HCMV genome [5]

Despite the diversity of genomic sequences of viruses belonging to the family Herpesviridae, there are conserved fragments (core genes) responsible for the basic metabolic and structural functions [5]. Genes specific to a group of viruses are not relevant in in vitro replication but play a role in the immune response. The sequence analysis with the genome of clinical isolates of HCMV revealed the presence of 70 glycoproteins, but in a laboratory strain AD169 only 57 were found. The structural glycoproteins are divided into two classes, i.e. common for the whole family ( $\mathrm{gB}, \mathrm{gH}, \mathrm{GL}$, $\mathrm{GM}, \mathrm{gN}$ ) and specific glycoprotein (having no resemblance to those of other herpesviruses). In contrast to the conservative glycoproteins, specyfic glycoproteins do not play a role in the replication of CMV cultures of fibroblasts in vitro, but are responsible for the tropism and pathogenicity of the virus [2]. 
The virus enters the cell by attachment virus glycoproteins of the lipid bilayer to the host cell membrane receptors. The capsid with the genetic material penetrates into the cytoplasm, then migrates to the nucleus. CMV has a slow replication cycle. Full replication cycle takes about 96 hours $[3,4]$.

During the lytic phase of infection, expression of immediate-early genes of the virus (IE) takes place, resulting in the formation immediate-early proteins that modulate cell metabolism and stimulate the expression of the early viral genes (E) [5]. At this stage, DNA replication occurs, whereupon early genes stimulate late viral gene expression. Late viral proteins are structural components of the virion and are involved in the formation of new virus particles. PostTranscriptional Gene Silencing (PTGS) viral early proteins in certain cell types, and is the cause of conversion into active infection in the latency stage. Latency is characterized by minimal expression of viral genes and inhibition of assembly and release of new progeny virions. Reactivation of latent infection is possible under the influence of various environmental factors. This leads to the development of disease and spread of the virus.

HCMV-infected cells also produce non-infectious particles that are similar to those of infectious virions comprise a shell, tegument and capsid proteins, but do not have DNA [2].

Viral matrix proteins, such as pp65, pp71, pp150 and pp28, play a role in all stages of the viral replication cycle; therefore, they constitute a target for new antiviral drugs [6]. PP 65 is a protein responsible for the modulation of the host immune response, both cellular and humoral immunity, and is the main target for cytotoxic T lymphocyte. The protein pp71 has a central role in the activation of the expression of immediate early genes and the onset of the lytic cycle of the virus; pp150 and pp28 are highly immunogenic and are involved in the formation and release of new virus particles. Furthermore, pp150, as well as pp65, is required for incorporation of the nucleocapsid into the viral particle. [6]

Epidemiology and pathogenesis of infection. The virus is widespread globally, and the primary infection is usually asymptomatic $[5,7]$. There is no seasonality of the disease. The prevalence of anti-CMV IgG depends on many factors, such as age, social and living conditions, geographical area, sexual activity (homo- and hetero-sexual infection). Research on the correlation of CMV infection with age revealed the existence of three periods of increased incidence of infections: early childhood, adolescence and the reproductive period.

The virus can be present in various secretions of an infected person, such as discharge from the oropharynx, urine, vagina, semen, milk, and blood components. The virus spreads by direct contact with an infected person, vertically from mother to foetus, and parenteral, i.e. blood transfusions, organ or bone marrow transplants [7]. Immunosuppression is a major risk factor for the development of severe primary infection. In this group of patients, the previous risk of cytomegalovirus pneumonia was $10-30 \%$ and approximately $80 \%$ mortality. But over the past several years this number has significantly decreased due to the use of prophylactic and / or therapeutic antiviral drugs to reduce the frequency of reactivation [8]. Transfer of infection by blood transfusion, associated with transfusions of packed red blood cells, platelet-rich plasma and concentrates of granulocytes. However, with transfusions of fresh frozen plasma and cryoprecipitate there is no such a risk of infection. The introduction of leukoreduction in blood donation centres has significantly reduced this risk [7].

The virus has an affinity for epithelial cells after infection and entering into the cells occurs the lytic replication $[2,5,7]$. The primary pathway of infection is the respiratory route or through sexual intercourse. Cytomegalovirus, like other herpesviruses, gives lasting settlement of the organism and its reservoir are mainly monocytes and polymorphonuclear leukocytes, where the virus is shed, even many years after the initial infection (present in the throat and urine).

The result of infection is seen substantially in larger cells, and the presence of characteristic intracellular inclusions surrounded by halo of low reflection, gives the appearance of the so-called. 'owl eyes'. Most primary infections are also recurrent in immunocompetent individuals and are asymptomatic.

The virus gets into the salivary gland, epithelial breast, prostate, endometrium, kidney tubules, and other organs, such as bone marrow and lungs. Therefore, it can be isolated from saliva, tears, breast milk, semen, cervical secretions, blood products, and urine [7].

CMV infection may be either latent (non-productive), lytic (productive), asymptomatic or symptomatic.

The important role of regulating the innate cellular immune cells such as natural killer (NK) and interferon [5]. As a result of activation of TLRs succeeding in increasing the production of cytokines and chemokines stimulate NK cells. However, the most important role is played by cytotoxic $\mathrm{T}$ lymphocytes CD4 + and CD8 +, which cause lysis of infected cells in the productive phase of infection, are involved in maintaining latent virus and preventing its reactivation. Therefore, people with a defect on the cellular response of $\mathrm{T}$ cells are a group at severe risk of infection. This is particularly important for patients undergoing organ transplant, or haematopoietic cell (HCT) and infected with HIV [8, 9, 10, $11,12,13]$. These people often become infected with primary or reactivated infection and re-infection (superinfection with a new strain of the virus). Polymorphism glycoprotein $\mathrm{B}(\mathrm{gB})$ determines viral tropism for different organs and tissues[14, 15]. Phylogenetic analysis of the region UL 144 showed high sequence diversity of clinical strains, suggesting a role in the infection of endothelial cells and leukocytes, it also demonstrated that genes UL128, UL130, UL131A play an important role in the ability of the virus to attack cells of epithelial and endothelial cells and leukocytes. Genotype protien $\mathrm{gB}$, such as $\mathrm{gB}$ 1or gGB2, is often associated with infections acquired in childhood and is different from the strains isolated from patients with HIV [16].

Clinical manifestations of CMV infection in immunocompromised patients include: prolonged fever over $38^{\circ} \mathrm{C}$ with (or without) leukopenia, hepatitis - there is a 2.5 -fold increase in transaminases; hyperbilirubinemia may occurs pancreatitis, gastrointestinal disorders, pain mainly present with fever, inflammation of the oesophagus, dysphagia, disorders that may be associated with Candida infection, interstitial pneumonia, inflammation of the heart muscle, bladder inflammation, inflammation of the retina particularly frequently observed in AIDS patients (patients without HAART in 60-71\%), inflammation of the brain - in patients with AIDS and after haematopoietic stem cell transplantation.

Cytomegalovirus is a very common cause of congenital infection in humans [7]. From the group of all newborns 
with congenital infections, approximately $10 \%$ with have symptoms of CMV. The most common symptoms of this disease are an enlarged liver and spleen, and petechiae on the skin. Congenital CMV infection can cause diffuse interstitial pneumonia with relatively severe prognosis. About $20 \%$ of children have disorders in the vision organ, such as inflammation of the retina and choroid, which in the case of macula lutea can lead to blindness, strabismus or optic nerve atrophy. They may be also negative symptoms from the central nervous system.

CMV infection is the most common post-transplantation that occurs within $1-6$ months after transplantation of solid organs, as well as bone marrow and haematopoietic stem cells $[8,9]$. Solid organ transplants carry a risk of infection with CMV in the range $8-50 \%$. Thoracic surgery treatments, such as transplant of lung, heart, or lung and heart, also have a high risk of infection $[13,14]$. Particularly vulnerable are seronegative recipients of seropositive donors (D + / R-). In these patients there is a high morbidity and mortality. (Tab. 1).

Table 1. The incidence of CMV infections in organ transplant recipients [by 15]

\begin{tabular}{lcc}
\hline \multicolumn{1}{c}{ Organ } & Infection (\%) & Disease (\%) \\
\hline Kidney & $8-32$ & 8 \\
\hline Heart & $9-35$ & 25 \\
\hline Liver & $22-29$ & 29 \\
\hline Lung (heart / lung) & $39-41$ & 39 \\
\hline Pancreas (kidney / pancreas) & 50 & 50 \\
\hline
\end{tabular}

Many studies point to the possibility of reactivation of CMV infection in immunocompetent patients, being in a serious condition because of clinical reasons, such as burns, trauma, sepsis, myocardial infarction $[9,10,11]$. In these cases, as a result of reactivation of infection in the lungs, cytomegalovirus pneumonia occurs.

Treatment and prevention. In immunocompetent individuals the disease is relatively mild and they do not require any medication. Pharmacological treatment with ganciclovir is used in immunocompromised patients (people with AIDS, organ transplant) whose disease is associated with the organ of vision or is life-threatening. The problem is the use of this drug during pregnancy, according to the classification FDA, it belongs to the $\mathrm{C}$ group of medicines. Valganciclovir (prodrug of ganciclovir) is an antiviral agent that also works effectively and is taken orally. Foscarnet can be administered to patients with CMV resistant to ganciclovir, although it is less tolerated than ganciclovir $[7,8,11]$.

The first trials of an anti-CMV were carried out in the 1970s. Elek and Stern developed a live attenuated vaccine, AD 169 , wherein the virus strain isolated from human lymphatic tissue was passaged 54 times in cultured human fibroblasts [17]. The vaccine was administered subcutaneously group of 26 seronegative volunteers. The injection was safe and well-tolerated. In 12 subjects of this group, while a local reaction to the vaccine occurred in only one person who had lymphadenopathy with lymphocytosis. A few years later, Neff et al. [18] conducted another clinical trial using AD169. The virus used in these studies was passaged an additional 5 times. Subcutaneous vaccine was administered to 24 volunteers. The immune responses, however, were of short duration.

Studies were also conducted using strains of CMV Towne, Toledo and Towne / Toledo. Plotkin et al. [19] isolated the Towne strain of CMV from the urine of 2-month-old infant with congenital cytomegalovirus. Virus has been passaged 125-times in tissue culture. The vaccine was given intramuscularly or intranasally and orally to 21 seronegative subjects. Seroconversion was achieved only after intramuscular administration. The vaccine introduced induced erythema and induration at the site of the injection in some individuals. Subsequent studies confirmed the absence of seroconversion with intranasal administration of the vaccine, as opposed to subcutaneous administration. Research has confirmed the ability of Towne to induce neutralizing antibodies $[20,21]$. The increase in antibody titres after vaccination with Towne was comparable to that which occurs with natural infection, it is also dose dependent. Antibody levels decreased during the year as opposed to a constant level in patients who have had natural infection. It was confirmed by the ability of Towne vaccine to induce a cellular response. A Towne vaccine study was also conducted in post-renal transplantation patients with high risk of cytomegalovirus infection. After vaccination, cellular and humoral immune response was observed. In seronegative recipients of kidneys from seropositive donors, the risk of developing severe disease was significantly reduced as a result of the vaccination $[19,21,22]$. Towne vaccine efficacy was also confirmed in studies with a not-attenuated, low passaged strain of CMV Toledo. The high degree of attenuation of the virus Towne is likely due to genetic mutations during intense passage. Towne strain contains numerous mutations in their genome, in contrast to the non-attenuated Toledo strain [23]. Specific mutations affecting the virulence are not known [21].

The next step in the study of HCMV vaccine was the creation of four independent chimeric vaccines containing genetically recombinant viruses, where regions of the notattenuated Toledo sequence were replaced by corresponding regions of the Towne genome [24]. The region Ul/b of the $\mathrm{CMV}$ genome responsible for encoding 19 proteins is present in HCMV, and is absent in strains after intense passages. The result is a recombinant Towne / Toledo virus which has an intermediate virulence between Towne and Toledo. Vaccines containing Towne / Toledo are safe and well tolerated. Viruses present in the vaccine are detected in the blood, saliva and urine of vaccinated individuals [22].

The Towne strain is currently being tested in a prime boost strategy where the attenuated vaccine virus is preceded by a DNA vaccine [25]. To increase the immunogenicity of the Towne vaccine, it is used with an adjuvant in the form of recombinant human IL $-12[25,26]$. Phase I clinical trial of this vaccine have been completed.

Subunit vaccines. Subunit vaccines use one or more specific proteins with an adjuvant to enhance the immune response of the organism. Glycoprotein B $(\mathrm{gB})$ is characterized by high immunogenicity and is unchanging. It is modified by removing the waterproof, transmembrane domain, and elimination of the internal space of proteolytic degradation. $[27,28]$. GB is used with aluminum hydroxide or MF59 as an adjuvant. MF59 is an emulsion of squalene in water. Squalene is polyunsaturated hydrocarbon, used as an 
adjuvant, for example, in an influenza vaccine. Research conducted with seronegative adults who were vaccinated with subunit vaccines, including $\mathrm{gB}$ with MF59 adjuvant in a study from 0-1-6 months. These people produced antibody levels comparable with those of natural infection with HCMV. Also, a different group of people were inoculated using glycoprotein $B$ vaccine with aluminum hydroxide in a similar scheme. However, this group produced significantly lower antibody titer. Specific antibodies IgG and IgA were detected in saliva and nasal secretions. Additional vaccination caused a rapid increase in antibody titer. Vaccination with gB/MF59 also induced lymphocyte proliferative response to gB and HCMV. The injection gave only slight pain at the site of application, and was well tolerated [29]. An ability of GB/MF59 to prevent children with congenital CMV among seronegative women is undergoing further studies, and phase II of clinical trials of the $\mathrm{gB} / \mathrm{MF} 59$ vaccine have been completed $[30,31,32]$. In the future, subunit vaccines may include, for example, $\mathrm{gH}$, $\mathrm{gM}, \mathrm{gN}, \mathrm{gO}, \mathrm{gL}$, and other HCMV antigens $[30,33]$.

Peptide vaccines. Peptide vaccines consist only of epitopes. Synthetic peptides of HCMV epitope pp65 induce T cellmediated cytotoxicity. This type of vaccine may be more useful in the treatment of rather than in preventing infection $[32,34]$.

Vector Vaccines. Vector Vaccines are another type of HCMV vaccines. Many viruses are used as vectors for present vaccine antigens. ALVAC is the attenuated strain of the canarypox virus (CNPV) and is one of the most frequentlyviral vectors used in HCMV vaccines [35]. The genome of this virus can produce a big exogenous DNA fragment, which allows a large selection of antigen gene or combination of genes. During the infection of human cells and expression of foreign antigens, its own genome does not replicate, thereby reducing the risk of vaccine-associated adverse effects. Protein gB was the first HCMV antigen used for expression with ALVAC. After promising results in animal research, human clinical trials were started. ALVAC-gB can be used in prime boost vaccines. HCMV seronegative adults receive two doses of ALVAC-gB on a 0 - 30 days, and then on the 90th day, a dose of live attenuated Towne vaccine [36]. This produced an immune antibody titer as high as in a natural infection. Similarly, when two doses of ALVAC-gB and a gB/MF59 dose of subunit vaccines, a high titer of immune antibodies was obtained and lymphoproliferative answer to HCMV antigen. Another type of vaccine is ALVAC, which allows expression of the viral protein pp65 [21]. Vector vaccine for HCMV is also a vaccine based on alphavirus vector carrying gene $\mathrm{gB}$ or fusion gene pp65/IE1, and is based on the vector MVA (modified vaccinia virus Ankara strain) also carrying the gB gene.

AVX601 vaccine. This is a two-component vaccine which has a carrier in the form of replicon Alphaherpesvirus, enabling the expression of HCMV glycoprotein B and pp65 (phosphoprotein 65) and IE1 (Immediate-early protein with a molecular mass of $72 \mathrm{kDa}$ ) [37]. The vaccine is safe, well tolerated, with mild to moderately-severe local reactivity, pain at the injection site, tenderness, redness, itching. Induced high levels of neutralizing antibody and specific antigen response of lymphocyte $\mathrm{T}$. It seems that this is a promising candidate for the prevention of congenital cytomegalovirus infection. The first phase of clinical trials on this vaccine has been completed, and the reaseach entered into phase II $[31,37]$.

DNA Vaccines. Preliminary and surprising research results indicate that the direct administration of an injection of purified DNA encoding specific antigens can induce protective immunity. This has led to the development of DNA vaccines for many pathogens, including CMV [31, 38]. They can induce both cellular and humoral immune response against CMV. The first DNA vaccine for HCMV contains a plasmid with gene for pp65. Another vaccine contained plasmids with the gene for pp65 to stimulate cytotoxic T-lymphocyte response and $\mathrm{gB}$ to stimulate the humoral immune response (bivalent DNA vaccine). VCLCB01 is a DNA vaccine with plasmids ncoding pp65 and gB. Clinical trials of this vaccine are now in phase II $[21,32$, 38]. The vaccine ASP0113 (TransVax), based on the same immune response pathways, are already at the stage of phase III trials. The study is a randomized, placebo-controlled, double-blind trial. The study group consisted of 500 patients undergoing haematopoietic cell transplant (HCT). Previous phases have produced very promising results of action of this vaccine [39]. VCL-CT02 / Towne is a prime booster vaccine consisting of a trivalent vaccine with DNA with plasmids encoding pp65, IE1 and gB. It is in phase I of clinical trials $[21,40]$. Vaccination of mice with a DNA vaccine using the $\mathrm{gB}$ gene and the gene for type I interferon resulted in a greater resistance to MCMV, compared to mice that received a DNA vaccine only for the $\mathrm{gB}$ gene. It is interesting that the DNA vaccine with gene for type I interferon reduces the severity of the MCMV infection [40, 41, 42].

Vaccination in the prenatal period. An important aspect is the prevention of congenital infection. Levinton at al [43] in his research focused on the study of the vaccine V545 in preventing transplacental transmission CMV infection in guinea pigs. The vaccine was created through the use of mutagens in guanosyl phosphoribosyl transferase (gpt) to remove viral gene GP1, which is responsible for the recognition of surface receptors on host cells. Also added to the genome were plasmids such as pKTS 107, pKTS 534, pKTS 536, among others. There was thus obtained an attenuated strain of the virus GP CMV MIP. Females were immunized twice at an interval of 3 weeks; then, the vaccinated animals were mounted by seronegative males (similarly conducted with the unvaccinated control group). After the confirmation of pregnancy, the females were infected with a virulent strain of CMV (salivary gland-derived GPCMV workpool), about four weeks before the birth. The test results (ELISA) show that the V545 vaccine had a significant impact on reducing the maternal viral load. After clinical examination, the protective effect of the vaccine against congenital CMV infection was noted in newborn guinea pigs. Animals born from vaccinated mothers had a higher birth weight and lower percentage of intrauterine death.

Promising results were obtained in phase II clinical studies among the subunit vaccines using recombinant HCMV gB protein in combination with the adjuvant MF59 for seronegative women in a prenatal infection control [44].

Alternative paths of development of vaccines. The following vaccines were tested in preclinical studies and in animal 
models: vector vaccine with recombinant vesicular stomatitis virus that transferred the gB gene, based on the vector MVA (modified vaccinia virus Ankara strain) also carrying the $\mathrm{gB}$ gene, and replication-deficient adenovirus-vectored vaccines. The tests were also carried with genetically modified plants (such as rice), their genome changed to induce an immune response in humans [45]. The new type of vaccine uses a genetically modified bacteria, created by expression in the modified bacteria proteins that are present on the surface of the pathogenic virus. These vaccines would include only fragments of the surface proteins of the virus which are placed in bacteries that normally exist in humans, e.g. Escherichia coli. Bacterial artificial chromosomes (BACs) are based on natural plasmids $\mathrm{F}$ of Escherichia coli, and can be used as a DNA vaccine. One chromosome is able to take fragments of $300 \mathrm{~kb}$. This type of vaccine may provide adequate viral genes and therefore induce a broad immune response to the virus [46].

Dense bodies (DBs) are complex, non-infectious particles produced by HCMV-infected cells. Through research on this issue, there is a new opportunity to create an effective vaccine against CMV. Their high immunogenicity by inducing cellular and humoral responses has been confirmed even in the absence of adjuvant [47].

\section{CONCLUSIONS}

Because of the very wide spread of CMV in the human population, and the various ways of spreading the infection, prevention of cytomegaloviral disease is limited. The only thing that can be done is to limit the spread of the virus by using basic hygiene guidelines for the prevention of influenza and other influenza-like illness. It seems that the ideal way to prevent the spread of CMV infections are vaccinations. The time between $2-6$ months of age is seems to be the optimal time for the implementation of the first vaccination. HCMV vaccination strategy should include first vaccinations before one year of age, and then another at the age of 10-11 years [48]. Vaccination would be particularly significant for females before the onset of puberty. This would reduce the risk of congenital CMV infection in the foetus and newborn. Recent studies have shown the safety of vaccines for both mother and as a child [49]. Another group of people for whom the benefits of the vaccine would be particularly significant are immunosuppressed patients. The presented study has shown the various activities that have existed during about 40 years work on the development of safe and effective vaccines, but to-date have not yet achieved the desired effect. However, ongoing research and clinical trials brings us closer to attaining a safe and effective vaccine. High hopes are attached to the TransVax vaccine which is the closest to being introduced into the pharmaceutical industry.

\section{REFERENCES}

1. Riley HD. History of the cytomegalovirus. South Med J. 1997; 90(2): 184-90.

2. Goodrum F, Cavines K, Zagallo P. Human cytomegalovirus persistence Cell Microbiol 2012; 14(5): 644-655.

3. Davison AJ, Dolan A, Akter A, Addison C, Dargan DJ, Alcendor DJ et al. The human cytomegalovirus genome revisited: comparison with the chimpanzee cytomagolovirus genome. J General Virol. 2003; 84: 17-28. J Gen Virol. 2003; 84(1): 17-28.
4. Shikhagaie M, Mercé-Maldonado E, Isern E, Muntasell A, Albà MM, López-Botet $\mathrm{M}$ et al. The human cytomegalovirus-specific UL1 gene encodes a late-phase glycoprotein incorporated in the viron envelope. J Virol. 2012; 0: 4091-4101.

5. Boeckh M, Geballe AM. Cytomegalovirus: pathogen, paradigm and puzzle. J Clin Invest. 2011; 12(5): 1673-1680.

6. Tomtishen JP. Human cytomegalovirus tegument proteins (pp65, pp71, pp150, pp28). Virol J. 2012; 9: 22-28.

7. Boppana S, Fowler KB. Persistence in the population: epidemiology and transmission. In: Arvin A., Campeadelli-Fiuwe g., Mocarski E. et al. Human Herpesviruses: Biology, Therapy and Immunology. Camridge University Press 2007.

8. Durlik M. Zakażenie wirusem cytomegalii u biorców przeszczepów narządowych. Nefrol Dial Pol. 2009; 13: 157-163.

9. Furmańczyk A, Durlik M. Nawrotowa choroba CMV o ciężkim przebiegu u pacjenta po przeszczepie nerki - opis przypadku. Forum Nefrol. 2010; 3(3): 169-173.

10. Limaye AP, Boeckh M. Cytomegalovirus (CMV) in critically-ill patients: pathogen or bystander? Rev Med Virol. 2010; 20(6): 372-379.

11. Cook ChH, Trgoccich J. Cytomegalovirus reactivation in critically ill immunocompetent hosts: A decade of progress and remaining challenges. Antiviral Res. 2011; 90(3): 151-159.

12. Melnick M, Sedghizadeh PP, Allem CM., Jaskol T. Human cytomegalovirus and mucoepidermoid carcinoma of salivary glands: Cell-specific localization of active viral and oncogenic signaling proteins is confirmatory of causal relationship. Exp Mol Pathol. 2012; 92:118-125.

13. Gerna G, Lilleri D, Chiesa A. et al. Virologic and immunologic monitoring of cytomegalovirus to guide preemptive therapy in solidorgan transplantation. Am J Transplant. 2011; 11: 2463-2471.

14. Hammond SP, Martin ST, Roberts K, et al. Cytomegalovirus disease in lung transplantation: impact of recipient seropositivity and duration of antiviral prophylaxis. Transpl Infect Dis. 2012; 0: 1-8.

15. Snydman DR, Limaye AP, Potera L, Zamora MR. Update and Review: Stae-of-The-Art Management of Cytomegalovirus Infection and Disease Following Thoracic Organ Transplantation. Transplantation 2011; 43: $1-17$.

16. Vogel JU, Otte J, Koch F. Role of human cytomegalovirus genotype polymorphisms in AIDS patients with cytomegalovirus retinitis. Med Microbiol Immunol. 2013 Feb;202(1):37-47

17. Elek SD, Stern H. Development of a vaccine against mental retardation cased by cytomegalovirus infection in utero. Lancet 1974; 1: 1-15.

18. Neff BJ, Weibel RE, Buynak EB, McLean AA, Hilleman MR. Clinical and laboratory studies of live cytomegalovirus vaccine Ad-169. Proc Soc Exp Biol Med. 1979;160(1): 32-37.

19. Plotkin SA, Higgins R, Kurtz JB. et al. Multicenter trial of Towne strain attenuated virus vaccine in seronegative renal transplant recipients. Transplantation 1994; 58(11): 1176-1178.

20. Adler SP, Starr SE, Plotkin SA, et al. Immunity induced by primary human cytomegalovirus infection protects against secondary infection among women of childbearing age. J Infect Dis. 1995; 171(1): 26-32.

21. Schleiss MR. Cytomegalovirus vaccine development. Curr Top Microbiol Immunol. 2008; 325: 361-382.

22. Zhong J, Khanna R. Vaccine strategies against human cytomegalovirus infection. Expert Rev. Anti Infect Ther. 2007; 5(3): 449-459.

23. Gil A, Shen S, Coley S, Gibson L, Diamond DJ, Wang S, Lu S. DNA vaccine prime followed by boost with live attenuated virus significantly improves antigen-specific $\mathrm{T}$ cell responses against human cytomegalovirus. Hum Vaccin Immunother. 2013; 9(10).

24. Heineman TC, Schleiss M, Bernstein DI, et al. A Phase 1 study of 4 live, recombinant human cytomegalovirus Towne/Toledo chimeric vaccines. J Infect Dis. 2006; 193(10): 1350-1360.

25. Jacobson MA, Adler SP, Sinclair E, et al. A CMV DNA vaccine primes for memory immune responses to live-attenuated CMV (Towne strain). Vaccine. 2009; 27(10): 1540-1548.

26. Jacobson MA, Sinclair E, Bredt B, et al. Safety and immunogenicity of Towne cytomegalovirus vaccine with or without adjuvant recombinant interleukin-12. Vaccine. 2006; 24(25): 5311-5319.

27. Pass RF, Zhang C, Evans A, et al. Vaccine prevention of maternal cytomegalovirus infection. N Engl J Med. 2009; 360(12): 1191-1199.

28. Pass RF. Development and evidence for efficacy of CMV glycoprotein B vaccine with MF59 adjuvant. J Clin Virol 2009; 46(4): 73-76.

29. Griffiths PD, Stanton A, McCarrell E, Smith C, Osman M. Cytomegalovirus glycoprotein-B vaccine with MF59 adjuvant in transplant recipients: a phase 2 randomised placebo-controlled trial. Lancet. 2011; 377(9733): 1256-1263.

30. Bernstein DI. Vaccines for cytomegalovirus. Infect Disord Drug Targets. 2011; 11(5): 514-525. 
31. Sung H, Schleiss MR. Update on the current status of cytomegalovirus vaccines. Expert Rev Vaccines 2010; 9(11): 1303-1314.

32. Chiurchiu' S, Calo' Carducci FI, Rocchi F, Simonetti A. Is HCMV vaccine an unmet need? The state of art of vaccine development. Int J Immunopathol Pharmacol. 2013; 26(1): 15-26.

33. Loomis RJ, Lilja AE, Monroe J, Balabanis KA, Brito LA. Vectored codelivery of human cytomegalovirus $\mathrm{gH}$ and $\mathrm{gL}$ proteins elicits potent complement-independent neutralizing antibodies. Vaccine 2013;31(6) 919-926.

34. Paston SJ, Dodi IA, Madrigal JA. Progress made towards the development of a CMV peptide vaccine. Hum Immunol. 2004; 65(5): 544-549.

35. Adler SP, Plotkin SA, Gonczol E, Cadoz M, Meric C, Wang JB. A canarypox vector expressing cytomegalovirus (CMV) glycoprotein $\mathrm{B}$ primes for antibody responses to a live attenuated CMV vaccine (Towne). J Infect Dis. 1999; 180(3): 843-846.

36. Minke JM, Fischer L, Baudu P, Guigal PM, Sindle T, Mumford JA, et al. Use of DNA and recombinant canarypox viral (ALVAC) vectors for equine herpes virus vaccination. Vet Immunol Immunopathol. 2006; 111(1-2): 47-57.

37. Bernstein DI, Reap EA, Katen K. et al.: Randomized, double-blind, Phase 1 trial of an alphavirus replicon vaccine for cytomegalovirus in CMV seronegative adult volunteers. Vaccine. 2009; 28(2): 484-493.

38. Hartikka J, Bozoukova V, Morrow J, Rusalov D, Shlapobersky M. Preclinical evaluation of the immunogenicity and safety of plasmid DNA-based prophylactic vaccines for human cytomegalovirus. Hum Vaccin Immunother. 2012; 8(11): 1595-1606a

39. Riedmann EM. Human vaccines \& immunotherapeutics: news. Hum Vaccin Immunother. 2013; 9(4):722-72.
40. Temperton NJ. DNA vaccines against cytomegalovirus: current progress. Int J Antimicrob Agents. 2002; 19(3): 169-172.

41. Kharfan-Dabaja MA, Boeckh M, Wilck MB, Langston AA, Chu AH. A novel therapeutic cytomegalovirus DNA vaccine in allogeneic haemopoietic stem-cell transplantation: a randomised, double-blind, placebo-controlled, phase 2 trial. Lancet Infect Dis. 2012; 12(4): 290-299.

42. Liu MA, Ulmer JB. Human clinical trials of plasmid DNA vaccines. Adv Genet. 2005; 55: 25-40.

43. Leviton M, Lacay J, Choi K, Hernandez-Alvarado N, Wey A, Schleiss M. An Attenuated Cytomegalovirus Vaccine with a Deletion of a Viral Chemokine Gene Is Protective against Congenital CMV Transmission in a Guinea Pig Model. Clin Dev Immunol. 2013; 906-948.

44.Lipińska A, Bieńkowska-Szewczyk K. Nowe szczepionki przeciw herpeswirusom i wektory herpeswirusowe w terapii człowieka. Post Mikrobiol. 2010; 49(3): 199-207(in Polish).

45. Khanna R, Diamond DJ. Human cytomegalovirus vaccine: time to look for alternative options. Trends Mol Med. 2006; 12: 26-33.

46. Plotkin S, Plotkon B. Cytomegalovirus vaccines. In: Reddchase M. Cytomegaloviruses from molecular pathogenesis to therapy. Coister Academic Press 2012.

47. Ahlqvist J, Mocarski E. Cytomegalovirus UL103 controls virion and dense body egress. J Virol. 2011; 85(10): 5125-5135.

48. Azevedo RS, Amaku M. Modelling immunization strategies with cytomegalovirus vaccine candidates. Epidemiol Infect. 2011; 139(12): 1818-1826.

49. Adler SP. Immunization to prevent congenital cytomegalovirus infection. Br Med Bull. 2013; 107: 57-68. 